\title{
25
}

\section{Benchmarking Techniques}

\author{
Dr.-Ing. Kai Mertins, Dipl.-Ing. S. Kempf, Dipl.-Ing. G. Siebert \\ Fraunhofer-Institute for Production Systems and Design Technology (IPK)
}

\section{Definition of Benchmarking from Our Point of View}

Benchmarking is a continuous process during which services and specially processes and methods of operational functions throughout several companies can be compared /Camp89/. With that differences to other companies as well as reasons for these differences shall be pointed out and possible improvements shall be determined. The comparison regards companies which control the methods and processes to be examined very well.

\section{Benchmarking techniques}

\subsection{General}

Soon it was recognized that it was not enough to examine only products as explanation for the different types of costs of the company, but that a fundamental knowledge of the single activities within the added value chain is a necessary assumption. For this purpose methods and tools are needed.

Benchmarking is the device which turns the unstructured process of a permanent improvement into an objective action plan. Benchmarking starts when problems are identified and concentrates on detecting kernel problems to improve the current practice.

For this purpose several techniques are available which partly complement respectivelyand/or base on each other. The selection of the technique is mainly determined by the aim of the increase of added value. For Benchmarking it is important to understand which advantages are to be expected before expensive ressources are invested. 


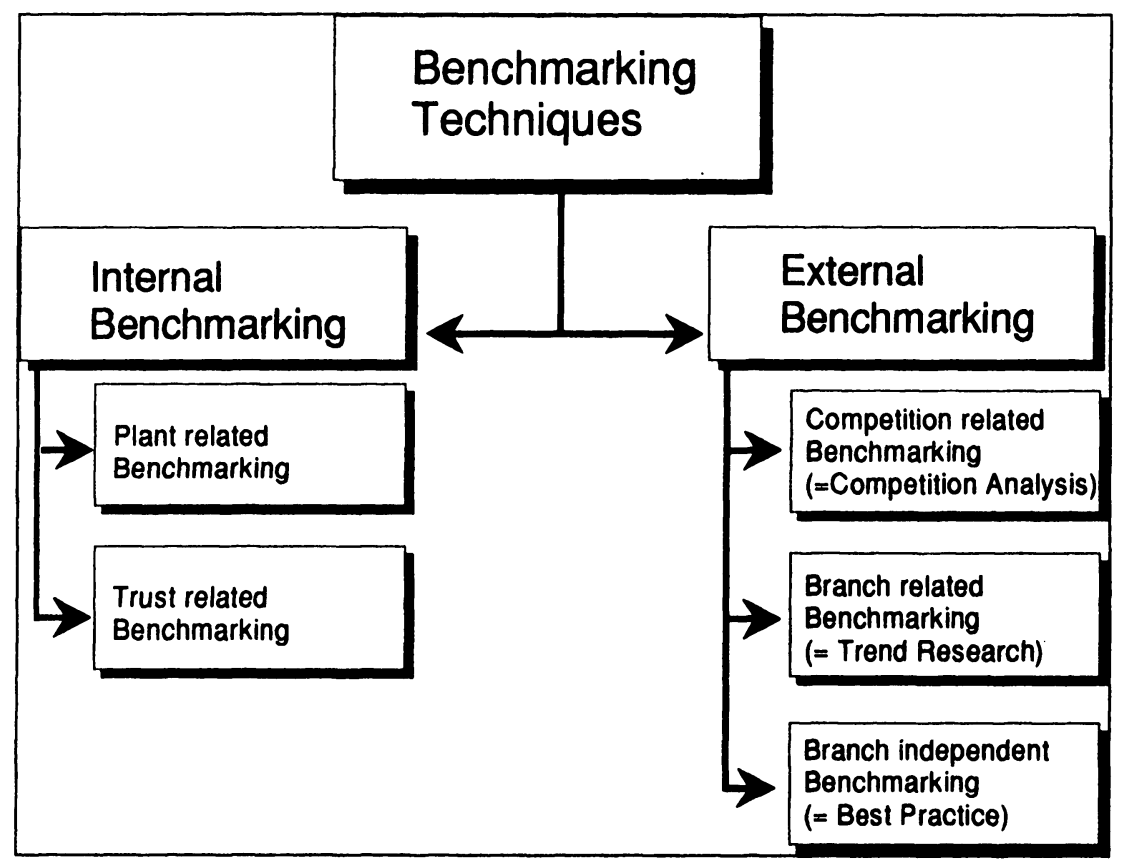

Figure 1: Benchmarking Techniques

\subsection{Internal Benchmarking Techniques}

Internal Benchmarking is the simpliest form of Benchmarking because no external limits have to be considered. With internal Benchmarking organizations try to learn from their branches, departments and sistercompanies. During these studies similar processes are examined and compared throughout different areas to obtain detailed information about the provided performance potential. The implementation and data access are easy, but the success regarding a performance increasing change is low because units belonging together have the tendency to submit to cultural and organizational standards. At the internal Benchmarking the look of the management is turned inside before it is turned outside. Current operating sequences and practices shall be registered objectively and shall be understood. This way the necessary details to concentrate the study on the aspects are obtained.

\subsubsection{Plant Related Benchmarking}

Inside of a company it is possible to find similiar processes which can be compared. Those processes are signed by technological, organizational and personal influences. 


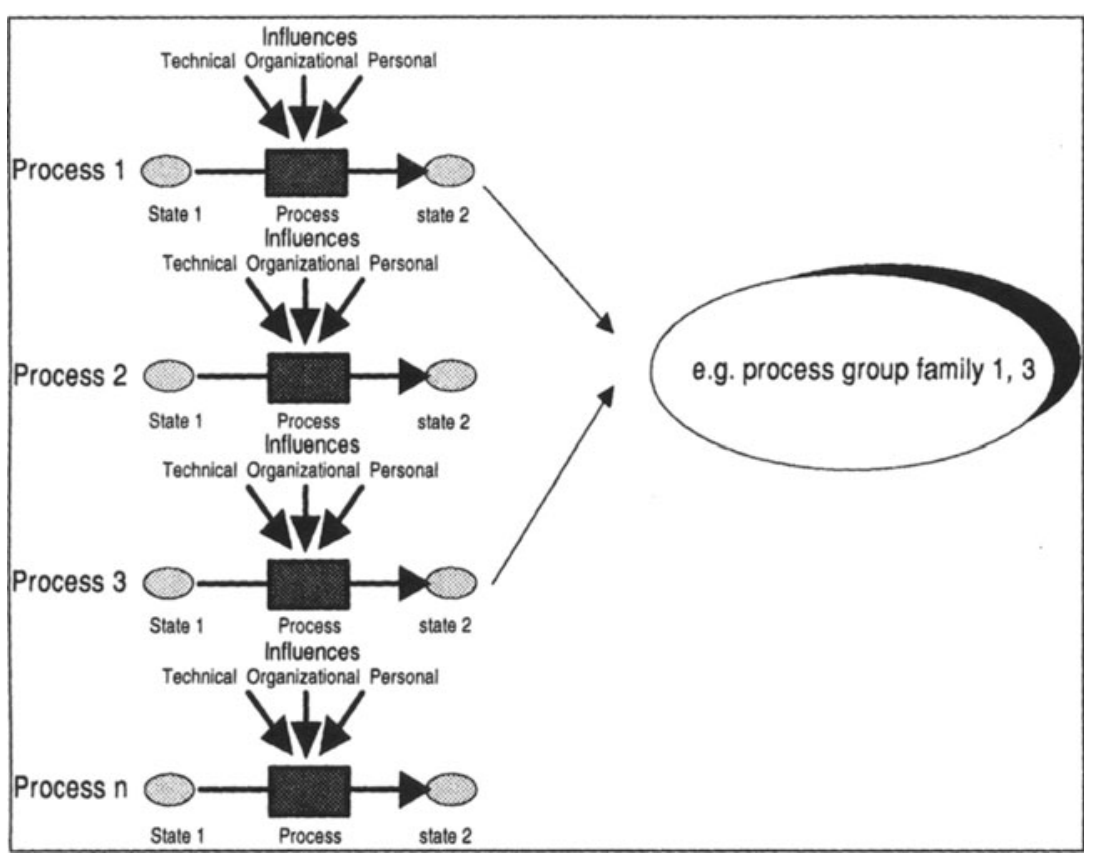

Figure 2: process group family

In this case it is possible to compare those processes by building similar process groups. This is a very specific way of benchmarking.

Definition of a process: A general mark of processes is according to DIN 19222 the mutual influence of operation within a system by which in their total amount matter, energy or information is transferred or stored. Processes are marked by a sequence of activities which again characterized by measurable inputs and outputs and can be part of a higher level processes. Additionally processes have to fulfil following four requirements:

Effectiviness: Processes have to fulfil the tasks and targets given to them.

Efficiency: Processes must fulfil their tasks with a minimum of expenditure.

Ability to test and control: The state of a process should be known at any time and there must be the possibility to make corrections.

Adaptability: Processes must be designed in a way that it can be reacted on changes in their surrounding.

\subsubsection{Trust Related Benchmarking}

This technique is used for the comparison of several plants within one company. Equal fields are examined, like e.g.: design, manufacturing etc..

This function oriented proceeding is the siemliest and best way to apply own ideas and capacity. Additionally a process is started through this channel of fair competition between the plants that builds the absolute assumption for continous improvement especially in connection with the external Benchmarking. 


\subsection{External Benchmarking Techniques}

Benchmarking is a technique for looking outside where at the practices of the own company are compared with the external practices. Comparison means that there must be a basis line of similarities. Only similar things can be compared each other. Therefore it is necessary to recognize one's own operations and processes. Only then a valid comparison and the identification of improvement chances are possible. The ideal case is that several companies should take part in such a study,

to minimize the costs,

$\square$ for easier data collection,

$\square$ the results can be used on a broad basis.

\subsubsection{Competition Analysis}

The competitions analysis can be provides information about the current and future market activities of the competitors, their strenghnesses and weaknesses as well as their possible reaction to specific behaviors. It also allows the comparison with companies which control the respective company activities in an excellent way, but it doesn't look beyond surroundings of the competitors.

An important difference between a pure competition analysis and Benchmarking is that functions and services are always measured from the best. While the exclusive comparison with the competitors only makes possible an equal footing with them, the comparison with the best permits to get ahead of the competitors.

Definite functions are often solved in a better way by non-competitors than within equal companies because of the special facilities of the companies.

Branch blindness and the delimit from competitors clearly make a further development more difficult. Benchmarking makes it possible to orient oneself on the best by the to noncompetitors and to learn by that how these companies manage to be ahead in definite fields.

\subsubsection{Branch Related Benchmarking}

Branch related Benchmarking goes beyond the mere comparison of two firms and emphasizes on the search for trends. So it examines the efficiency of a definite function throughout the branch. For that purpose it is necessary to examine a much larget group of companies than it is the case at the competion related Benchmarking.

It is not restricted to the performance of two or three others who sell the same products in about the same market but it is founded on broaded fields. Branch related Benchmarking looks for trends instead of competition positions and serves for the performance analysis of subsystems. Where at the limits to the competition related Benchmarking are fluent because up today there is no answer to the question where a straight study ends and the trend research begins.

\subsubsection{Branch Independent Benchmarking (Best Practice)}

The key to a long term success in competion is not equality but superiority. One wants to overtake the presently existing best practice an then get ahead of it. Therefore the final form of Benchmarking is aligned with the best. It searches throughout a spectrum of branches for new, innovative practices, independent from their source.

Benchmarking from the best of the class is based on the conviction that the process of added value is based on similar characteristics throughout many different institutions. Therefore the aim is to find the best of the best practices and to use them to alter innovatively 
the existing practices within the own organization. To define success by external criteria means to develop an idea of best performance and to heighten the company to a new performance level.

Only by that it is possible to leave the competitors further behind and to reach world's class.

\section{Benchmarking Problems}

Benchmark solutions are always only as good as the respective Benchmarking partner, regardless wether internal or external Benchmark is concerned. From that the range of problems which every Benchmarket is faced with derive.

How do I find the best suited Benchmark partner for my target. This Benchmark partner must meet a number of criteria to be worth the effort for the examination, like e.g.:

Are the operating sequences comparable?

Are the range of problems comparable?

Is the Benchmark partner "best of class" in the field of the problem?

If there is no possible Benchmarking partner known yet who meets the demanded criteria, a new partner must be found. But for external Benchmarking following problems appear at getting the necessary information:

$\square$ Information about companies often only exist as branch oriented sorting.

Further sorting criteria are often only the size of the company and turnover.

There are no information about company specific strenghnesses or weaknesses available.

Due to this hierarchical information structure the target group soon is limited to the members of the own branch. Information about branch external companies who possibly could be a very well suited Benchmarking partner thus quickly get out of sight and it takes a lot of effort to get them.

For the internal Benchmarking the range of problems are slightly different:

$\square$ the process sequences are not documented and so

$\square$ the process sequences are not comparable.

But for a process oriented Benchmark examinations the comparability of process sequences are of special importence because otherwise very similar process sequences from other company functions could be overlooked and thus be cancelled as Benchmark partner.

\section{Our Solution}

If it is managed to sort company information not any longer in a hierarchical way, but to show all relevant characteristics simultaneously, then it is possible to remove the branch spectacles completely. By that the procedure of finding information can be quickly performed without taking the risk of overlooking important Benchmarking partners.

An approach to solutions of this kind of problems can be the arrangement of company information according to characteristics on a company independent abstract level. As an example a comparison between the electronics industry with the insertion of circuit boards and the food industry with the insertion of chocolate in boxes can be used. These processes are similar in order and control and therefore can belong to one company group. So this arrangement provides branch independent company information that is the ideal basis for the search for Benchmarking partners. Following targets can be achieved by the branch independent arrangement of company information:

$\square$ save finding of the best suited Benchmarking partner and

$\square$ reduction of the time expenditure to find this Benchmarking partner. 
A similar approach to a solution could be used for the internal Benchmarking. If it is managed to document and classify the process sequences in a way that they can be aggregated in groups of similar process sequences by adequate tools then it is possible to use the potentials offered by Benchmarking also for company internal comparisons, see also figure 2.

In the sense of KVP or Kaizen Benchmarking will be completed as an instrument which managers will use in daily life just like using the telephone. Initiation to changes; instruction of strategy recommendations that must be hardly acquired then; continuous Benchmarking circuits, report of an alteration plan.

The goal of Benchmarking is to reach a competition advantage and to hold this competitive position /Christ/.

\section{References}

/Camp89/

Camp, Robert: Benchmarking: The Search for Industry Best Practices that Lead to Superior Performance, Milwaukee, Wisconsin 1989.

/Christ/

/DIN 19222/

Charles Christ, President of the Xerox Reprographics Group, 1980.

/From92/ Fromm, H.: Das Management von Zeit und Variabilität in

Deutsche Elektrotechnische Kommission im DIN und VDE (DKE): DIN 19222, Berlin: Beuth Verlag, März 1985.

\section{Curriculum Vitae of Dr.-Ing. Kai Mertins}

Dr.-Ing. Kai Mertins was born 1947 in the Federal Republic of Germany. After studying Control theory in Hamburg and Economy together with production technology at the Technical University of Berlin, he became member of the scientific staff of the University Institute for Machine Tool and manufacturing Technology (IWF), Berlin/FRG. Since 1983 he had been head of the department "Production Control and Manufacturing Systems" at the Fraunhofer-Institute for Production Systems and Design Technology (IPK), Berlin/FRG, where he has been Director for Planning Technology (President: Prof. Dr. h.c. mult. Dr.-Ing. G. Spur) since 1988 . He has more than 15 years experience in design, planning, simulation and control of flexible manufacturing systems (FMS), manufacturing control systems (MCS), shop floor control systems, and computer integrated manufacturing (CIM). He was General Project Manager in several international industrial projects and gave lectures and seminars at the Technical University Berlin, Polytechnic Nottingham/UK, Czech Republic, Indonesia and China. Special field of interest: Manufacturing strategy development, planning for production systems, shop floor control and simulation.

\section{Dipl.-Ing. Stefan Kempf}

Born 1963.

Dipl.-Ing. in Mechanical Engineering at the Technical University of Berlin, Germany.

Mr. Kempf is working for the Fraunhofer-Institute for Production Systems and Design Technology (IPK) at the department Planning Technology since 1990.

His working areas are the planning of production systems, management consulting, application of grouptechnology, management of teams working on large software projects and developing software. 


\section{Dipl.-Ing. Gunnar Siebert}

was born 1963 and after studying Industrial Engineering at the Technical University of Berlin he is working for the Fraunhofer-Institute for Production Systems and Design Technology (IPK), Berlin/FRG at the department Planning Technology. Since 1992 he is the Manager of the CIM Technology Transfer Center in Berlin/Brandenburg.

His working areas are management consulting, establishing logistics systems, introduction and establishing of CIM-Systems and benchmarking. 